\title{
Pré-tratamentos na produção de etanol de segunda geração
}

\author{
Eduardo De Rossi', Cleber Antonio Lindino², Kenia Gabriela dos Santos ${ }^{3}$, Reinaldo Aparecido Bariccatti \\ Paulo André Cremonez ${ }^{5}$, Jhonatas Antoneli ${ }^{6}$, Willian Cezar Nadaleti ${ }^{7}$ \\ 1,3,5,6 Mestrandos(as) em Engenharia de Energia na Agricultura, Universidade Estadual do Oeste do Paraná \\ ${ }^{2}$ Doutorado em química, Universidade Estadual do Oeste do Paraná \\ ${ }^{4}$ Graduado em Química, Bacharelado e Tecnológico, Universidade Estadual do Oeste do Paraná \\ ${ }^{7}$ Doutorando em Engenharia Ambiental, Universidade Federal de Santa Catarina
}

\begin{abstract}
Resumo
O aumento da produção de etanol sem expansão das áreas de plantio vem sendo estudado, no Brasil, mais intensivamente desde a iniciativa Proálcool. O biocombustível celulósico é considerado uma alternativa para expandir esta produção em larga escala, pois, não apenas possibilita a utilização do bagaço de cana, como também qualquer material lignocelulósico, e partes não comestíveis das plantas, além de que, o apelo ambiental viabiliza a produção em curto prazo. Um dos desafios é melhorar o processo de hidrólise da celulose e hemicelulose, sendo que a primeira apresenta uma estrutura quase cristalina e a segunda tem uma estrutura menos regular. O presente trabalho tem como objetivo relatar os principais pré-tratamentos utilizados com intuito de expor a celulose e a hemicelulose para a hidrólise (sacarificação). $\mathrm{O}$ grande desafio é devido à metabolização do polissacarídeo que gradativamente é dificultada à medida que aumenta complexidade de sua cadeia. A degradação da celulose trama uma rede de diversas possibilidades sendo possível até a produção de combustível para aviões a jato. Mesmo com o fato desta área de pesquisa ser recente, já existe fábrica piloto para tal produção e as primeiras refinarias instaladas entraram em funcionamento em 2011, contudo para que esta se difunda e ganhe mercado terá de competir com o poderoso mercado do petróleo.
\end{abstract}

Palavras-chaves: Energias renováveis; Biocombustível; Hidrólise; Celulose.

\begin{abstract}
The increasing in ethanol production without expansion of plantation areas has been studied in Brazil, more intensively since the Proálcool initiative. The cellulosic biofuel is considered an alternative to expand this production in large scale for it does not only allow the use of sugar cane bagasse, as well as any lignocellulosic material, and uneatable plant parts, besides, the environmental appeal enables the short term production. One of the challenges is to improve the process of hydrolysis of cellulose and hemicellulose, once that he first presents an almost crystalline structure and the second has a less regular structure. This paper aims to report the main used pre-treatments aiming to expose the cellulose and hemicellulose for hydrolysis (saccharification). The greatest challenge is due to the metabolization of the polysaccharide that is gradually impaired as the complexity of its chain increases. The degradation of cellulose weaves a network of several possibilities including even the fuel production for jet aircraft. Even with the fact that this area of research is recent, there is already such a pilot plant for production and the first refineries installed went into operation in 2011, however, in order to spread and gain markets it will have compete with the powerful oil market.
\end{abstract}

Keywords: Renewable energy; Biofuel; Hydrolysis; Cellulose. 


\section{INTRODUÇÃO}

A crescente preocupação com a elevação dos preços dos combustíveis fósseis e problemas ambientais, como o aquecimento global e saúde nas grandes cidades, tem sido alguns fatores que contribuem para a redução do consumo destes combustíveis. Entre os combustíveis alternativos têm-se os obtidos a partir da biomassa, e o etanol é um destes biocombustíveis que já faz parte da produção comercial brasileira em grande escala (Leite, 2007; World Watch Institute, 2006; Jr. Pereira, 2008)

O etanol, também chamado álcool etílico $(\mathrm{C} 2 \mathrm{H} 5 \mathrm{OH})$ é obtido por meio da fermentação dos açúcares encontrados em produtos vegetais (Bastos, 2007). Com a criação do Programa do Álcool, o PROALCOOL, lançado pelo governo brasileiro no ano de 1974 e implantado em 1975, o etanol como combustível, obtido por meio da cana-de-açúcar, passou a fazer parte do cenário comercial brasileiro. Atualmente o país é considerado um dos maiores produtores deste álcool do mundo e juntamente com os Estados Unidos correspondem a 89\% da produção global (Limayem e Ricke, 2012).

O clima brasileiro é um dos fatores que contribuem para esta produção de energia de biomassa (Marconato e Santini, 2008), e um dos problemas associados à produção de etanol é a elevada quantidade de bagaço de cana gerado no processo de produção. Desta forma tem-se buscado processos para o reaproveitamento deste resíduo de forma a melhorar a produção de etanol. A alternativa para minimizar este problema é contornada pela aplicação de mais de $90 \%$ do bagaço, que gerado na usina, para a produção de energia em sistema de cogeração, tornando a usina auto sustentável energeticamente (Conab, 2011).

Contudo, o bagaço de cana pode ter outro destino, sendo utilizado para obtenção de diversos produtos como a maximização da produção de etanol, o que possibilita a duplicação da produção da usina, sem que seja necessário aumentar as áreas de cultivo de cana-de-açúcar, muito menos necessita de que haja um aumento na produtividade por hectare. (Pereira, 2008; Trigueiro, 2013). Tornando-se necessários estudos econômicos da viabilidade da utilização dos materiais lignocelulósicos para produção de etanol (Bansal, 2013).

O combustível hoje denominado etanol de segunda geração apresenta vantagens quando comparado à gasolina e outros combustíveis derivados do Petróleo, pelo fato de ser considerado um combustível renovável. Estas vantagens poderão ser ampliadas, pelo aproveitamento da grande área de plantio já existente que o país apresenta, pois a quantidade de etanol produzida pode aumentar significativamente com a utilização do bagaço proveniente da quebra da lignocelulose. Essa maior produtividade pode garantir a continuidade da utilização do etanol, especialmente por apresentar produção extra e ter a capacidade de fazer a salvaguarda de quebras de safra, perdas sazonais, etc. (Sauer, 2007). Exemplo de quebra de safra ocorreu no ano de 2010, a falta de etanol nas bombas de combustíveis do país, o que aumenta a desconfiança do consumidor da mesma forma que ocorreu em 1989.

De acordo com a Petrobrás Biocombustível (2013), a produção de etanol de segunda geração a partir do aproveitamento de bagaço de cana já é realidade. O diferencial desta nova geração de biocombustível é que o uso do bagaço de cana aumenta $40 \%$ à produção de etanol em uma mesma área de plantação.

As matérias-primas para a produção de etanol celulósico (bioetanol) que podem ser utilizadas são madeiras, gramíneas, algas, qualquer material que contenha celulose ou hemicelulose. Estas são mais abundantes que as culturas alimentares e podem ser utilizadas sem causar interferência na economia alimentar (Basal et al., 2013). O etanol de segunda geração é aquele que necessita de hidrólise para liberação de açucares passiveis de redução biológica.

O bagaço de cana passa obrigatoriamente por um processo de hidrólise, em alguns casos também pelo pré-tratamento, e por final por um processo de fermentação, que é o mesmo utilizado para o etanol de primeira geração, salvo os casos em que de sacarificação e fermentação ocorrem em conjunto (Huber e Bruce, 2009).

O presente trabalho tem como objetivo relatar os principais pré-tratamentos utilizados permitindo a exposição da celulose e a hemicelulose para a hidrólise (sacarificação), que por sua vez podem ser feitas por rotas diferentes como as enzimáticas, ácidas, básicas, sofrendo influências da temperatura e do potencial hidrogeniônico. 


\section{PRODUÇÃO DE ETANOL UTILIZANDO O BAGAÇO DE CANA-DE-AÇÚCAR}

Com avanços observados na área de biotecnologia possivelmente a produção em escala comercial torne-se realidade, a custos competitivos partindo de matérias que contenham celulose e hemicelulose como o bagaço e a palha da cana-de-açúcar, principais matérias-primas utilizada para tal fim no Brasil. O etanol produzido no Brasil quando comparado a produção de outros países apresenta uma redução da emissão de dióxido de carbono e alternativas com melhor custo-efetividade além de reduzir a emissão de outros gases de efeito estufa (GEE). Tem o menor custo por tonelada de GEE evitado. Apenas pelo fato de utilizar o bagaço para aquecer as caldeiras nas indústrias sem nenhum tratamento no lugar do óleo diesel utilizado anteriormente já se tem dados de 33,2 milhões de toneladas equivalentes de $\mathrm{CO}_{2}$ em 2003, o que é o equivalente ao emitido pela Noruega no mesmo ano.

Os motores flex incentivaram em 2003 o retorno da utilização álcool como combustível de veículos leves, em larga escala, o que em comparação com a gasolina, emite $85 \%$ menos poluentes por litro, obviamente devemos levar em consideração que o álcool tem uma eficiência energética $30 \%$ menor quando comparada a gasolina, ou seja, para mesmos fins a emissão de dióxidos de carbono é mitigada, porém não tanto ao ponto de chegar a $85 \%$.

Segundo Seleghim e Polikarpov (2009), uma usina processando 500 toneladas por hora de cana de açúcar, pode produzir cerca de 45 mil litros por hora de etanol de primeira geração e aproximadamente 150 toneladas de bagaço que, atualmente, são integralmente convertidas em energia elétrica a uma taxa de 50MW.

Para Santos e colaboradores (2009), dois terços da massa da cana é fibra, biomassa não fermentável, necessitando de hidrólise para conversão de fibras em glicose, frutose, maltose e outros açucares fermentáveis e não fermentáveis e também inibidores da fermentação como o furfural. Na questão de aproveitamento de outras plantas, muitas nem detêm açúcar ou amido para a fermentação alcoólica, apenas celulose e hemicelulose, além da lignina, que no processo deve ser descartada ao máximo possível. A tecnologia de hidrólise enzimática é uma das mais promissoras na produção de etanol de segunda geração.

Perante Cerqueira e Cortez (2007), a fixação de $\mathrm{CO}_{2}$ ocorre por meio de fotossíntese, produz $1 \mathrm{~kJ}$ de energia no etanol a cada $0,12 \mathrm{~kJ}$ gastos de energia fóssil, ou seja, a cada unidade de energia fóssil utilizada gera-se cerca de oito unidades de energia renovável. O etanol tem a grande vantagem quando comparado à gasolina na questão de recuperação das emissões de dióxido de carbono emitidas em sua queima enquanto a gasolina que é um produto derivado do petróleo não tem um retorno ao petróleo, em ciclo de curto período.

\section{PRÉ-TRATAMENTO}

O estágio "pré-tratamento" é primordial para se obter uma conversão enzimática eficiente, elevar a área superficial da biomassa, a porosidade dos materiais, também sendo responsável pela hidrólise da hemicelulose (Hector, 2009). Hemicelulose é um polímero de carboidrato presente na matéria vegetal, tem como base principal de sua formação heteropolímero de pentoses, açúcares de cinco carbonos, como também xilose e a arabiose, hexoses como a glicose, manose e galactose e ácidos úricos (Aguiar et al., 2002).

Os pré-tratamentos podem ser realizados utilizando ácidos, bases, vapor, ou até mesmo a combinação entre eles, sabendo-se que o vapor de amônia é uma base. Dentre os vários métodos de pré-tratamento, os mais utilizados são Expansão da fibra em amônia (AFEX), hidrólise, Processo em Separado de Sacarificação (SHF) e Fermentação Simultâneas (SSF) (Sun e Cheng, 2005). O prétratamento separa a lignina da celulose e hemicelulose, os deixando disponíveis como demonstrado na Figura 1.

\section{EXPANSÃO DA FIBRA EM AMÔNIA (AFEX)}

Segundo Huber e Bruce (2009), uma técnica promissora que condiciona o material celulósico a condições extremas de $\mathrm{pH}$ e temperatura. O AFEX inicia-se a uma temperatura de cozimento da 
biomassa a $100^{\circ} \mathrm{C}$ em amônia concentrada sofrendo influência de pressão, quando esta é liberada a amônia evapora e é reciclada, então a biomassa é inserida seca e após o processo não encontrasse diluída em água, o que possibilita uma melhor hidrolise logo em seguida, além de a degradação da celulose ser mínima devido ao fato da não se tratar de um ambiente ácido ou de elevada temperatura, o que favorece para menor formação de pentoses.

O processo AFEX diminui o grau de cristalinidade, propicia a menor formação da pentose xilana e pode recuperar a amônia, mas não é efetiva quanto à destruição da lignina, necessitando de grandes quantidades de amônia no processo (Santos et al., 2012).

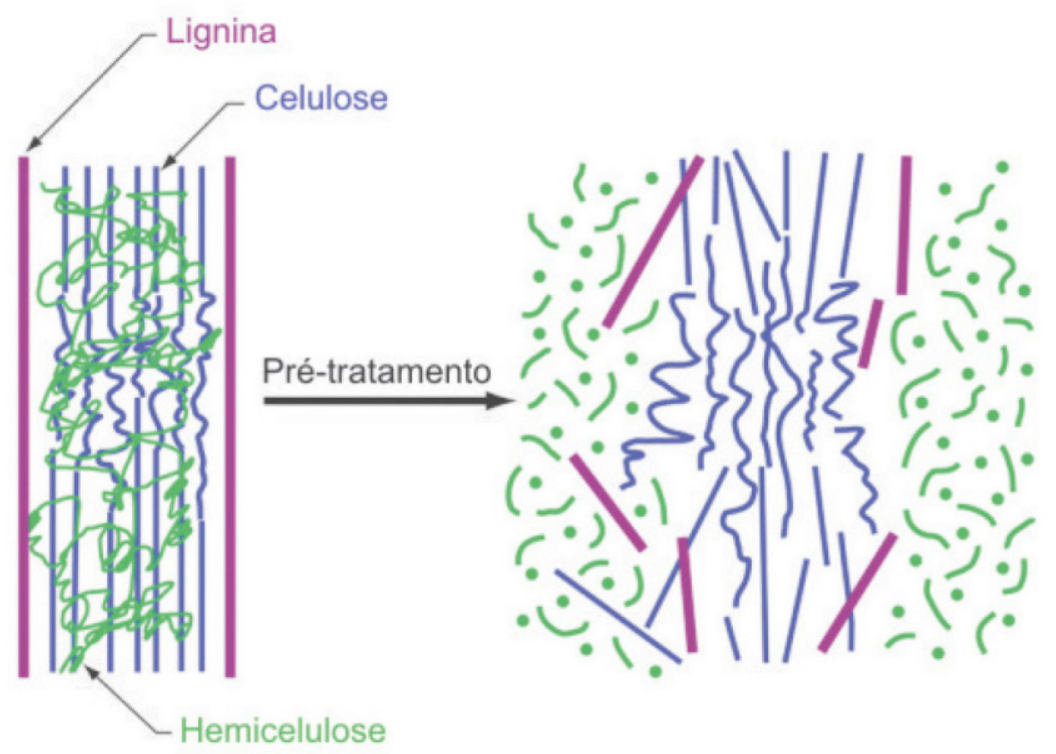

Figura 1. Alterações estruturais do complexo celulose-hemicelulose-lignina determinadas pelo pré-tratamento (Santos et al., 2012).

\section{HIDRÓLISE}

\subsection{Método de decomposição pela temperatura}

Perante Huber e Bruce (2009), os métodos de degradação da celulose através da temperatura são considerados: Baixos $\left(50\right.$ a $\left.200^{\circ} \mathrm{C}\right)$ produzem açucares, que podem ser fermentados e transformados em etanol; Altos $\left(300 \mathrm{a} 600^{\circ} \mathrm{C}\right.$ ) produzem bio-óleo $\mathrm{cru}$, que pode ser refinado para gasolina ou óleo diesel (biodiesel); Extremamente altos (acima de $700^{\circ} \mathrm{C}$ ), produzem gás, que pode ser convertidos em combustíveis líquidos.

É um modo de decomposição física sendo em pequena escala pode ser precedido de um moinho de bolas que inicialmente reduz a cristalinidade, porém possui alto consumo energético (Santos et al., 2012).

Os fatores que devem ser levados em consideração neste modelo de pré-tratamento é o percentual de amônia e água, tempo, temperatura e numero de tratamentos, além da velociadade de redução da pressão que deve ser o mais rapido possível. Operando em valores geralmente em torno de $90^{\circ} \mathrm{C}$ por 30 min, com cerca de $2 \%$ de amônia líquida, relação com massa seca da biomassa (Chiaramonti et al., 2012).

\subsection{DHR-DEDINI hidrólise rápida (ácida)}

Este tratamento tende a gerar elevadas quantidades de açúcares, obtidas da fração hemicelulósica (Baudel et al., 2006). Os ácidos mais empregados neste tratamento são $\mathrm{H} 2 \mathrm{SO} 4, \mathrm{HCl}, \mathrm{HF}, \mathrm{CH} 3 \mathrm{COOH}$ e HNO3.

Rossell (2002) utiliza um forte solvente da lignina, que é exatamente o agente restritivo da hidrólise, em altas temperaturas para facilitar o rápido acesso a celulose e hemicelulose, é um pré-tratamento e processo de hidrólise em união, o solvente da lignina, é forte e um ácido fraco, facilita o processo de hidrólise. A retirada do açúcar formado deve ser imediatamente após a hidrólise e resfriado para 
que não haja uma degradação deste carboidrato, principalmente em pentoses. Sendo logo em seguida neutralizado para estabilizar o açúcar obtido (Baudel et al., 2006).

\subsection{Hidrólise enzimática}

Enzimas especificas, como as celulases, são responsáveis pela hidrólise da celulose. São caracterizadas como um complexo de enzimas que possuem a capacidade de atuarem eficientemente (Arantes e Saddler, 2010).

Diversas bactérias podem ser utilizadas no pré-tratamento como Clostridium thermocellum e Bacteroides cellulosolvens, porém não alcançam elevada taxa de crescimento quando em celulose. Alguns estudos tem utilizado até mesmo fungos filamentos para a realização deste pré-tratamento (Duff e Murray, 1996; Martins, 2005).

Segundo Santos, Souto-Maior e Gouveia (2009), a celulose é uma estrutura que fornece o apoio estrutural a um vegetal de forma cristalina, é polissacarídeo de material rígido interconectado que sustenta o crescimento vertical da planta, este é o objetivo do tratamento, degradar pela ação da enzima até chegar à glicose. A glicose é um dos produtos da reação de sacarificação, a principal, e é obtida pela conversão da celobiose, com utilização da enzima $\beta$-glucosidase, a celobiose provém da quebra da celulose pela enzima celulase.

\subsection{Processo em SHF e SSF}

Na visão de Santos, Souto-Maior e Gouveia (2009), seria a produção de etanol lignocelulósico por hidrólise enzimática (sacarificação) e fermentação, tais processos podem ocorrer em separado ou simultaneamente. Processo em Separado (SHF) e Sacarificação e Fermentação Simultâneas (SSF), o primeiro tem melhor ação a uma temperatura sobre a sacarificação e a fermentação de $55^{\circ} \mathrm{C}$ e $30^{\circ} \mathrm{C}$ respectivamente. $\mathrm{O}$ microrganismo responsável pela fermentação necessita de condições de anaerobiose (isenta de oxigênio), já o segundo requer agitação de 150rmp, para a sacarificação em sua melhor eficiência, geralmente.

Ambos representam vantagens em diferentes aspectos, no SHF pode-se especificar temperaturas ótimas para a ação das enzimas e, a temperatura ótima para o fungo (Saccharomyces cerevisia), e no SSF a formação de glicose é acelerada e em maior quantidade, exatamente pelo fato de sua utilização em loco. Sendo assim maior o consumo dos produtos formados deslocando o equilíbrio para formação dos produtos (Santos et al., 2010).

SHF necessitaram no experimento de Santos et al. (2010), de 24 h de fermentação além das 48 de sacarificação, totalizando $72 \mathrm{~h}$. Enquanto o processo SSF necessita de apenas $16 \mathrm{~h}$ de pré-sacarificação e mais $24 \mathrm{~h}$ de fermentação e sacarificação simultâneas, resultando $40 \mathrm{~h}$. Os testes dos processos em união apresentaram em sua maioria uma maior produção em volume de álcool que o processo em separado. Além de corresponder a uma utilização de menores quantidades das celulases e $\beta$-glucosidase.

Devido à complexidade envolvida na tecnologia de transformação do bagaço em etanol através da sacarificação (hidrólise), torna-se necessário projetar e montar unidades-piloto, em uma escala intermediária, para se obter os dados de desempenho correlativos e otimizar o processo (Jr. Seleghim, 2006)

\section{CONCLUSÕES}

Torna-se notório em vários trabalhos a necessidade no desenvolvimento de leveduras apropriadas para condução da fermentação de hexoses, e no desenvolvimento de microrganismos que fermentem pentoses em etanol otimizando os pré-tratamentos do material celulósico visando à melhora da conversão enzimática, resultando em menos resíduos.

Com relação à sacarificação e fermentação deve-se analisar qual o modelo proporciona o maior custo benefício entre o SSF e o SHF, que o processo SSF pode apresentar uma maior produção de furfural, um inibidor de desenvolvimento do fungo responsável pela fermentação.

A seleção da matéria prima é uma decisão importante devido que a quantidade de lignina interfere negativamente no processo devido sua complexidade estrutural e isso pode representar aumento nos custos de produção. 


\section{REFERÊNCIAS}

AGUIAR, C.L.; MENEZES, T.J.B.; Conversão enzimática do bagaço de cana-de-açúcar. Biotecnologia e Ciência e Desenvolvimento, n.26, 2002.

BANSAL, A.; LLLUKPITIYA, P.; SINGH, S.P.; TEGEGNE, F. Economic competitiveness of ethanol production from cellulosic feedstock in Tennessee. Renewable Energy, v.59, p.53-57, 2013.

BASTOS, V. D. Etanol, alcoolquímica e biorrefinarias (2007). BNDES/Setorial, Rio de Janeiro, n.25, p.5-38. Disponível em: <http://homologa.ambiente.sp.gov.br/etanolverde/saibaMais/artigos/Producao/alcoolquimica.pdf $>$ Acesso em 06 dez. 2013.

BAUDEL, H. M.; ZAROR, C. Z.; ABREU, C. Catalytic hydrogenation of sugarcane bagasse dissolving pulp effluents over ru/c catalyst: an ecoefficient environmentally-friendly approach. In: XX SIMPÓSIO IBERO-AMERICANO DE CATÁLISE, Gramado-RS, 2006.

CHIARAMONTI, D.; PRUSSI, M.; FERRERO, S.; ORIANI, L.; OTTONELLO, P.; TORRE, P.; CHERCHI, F. Review of pretreatment processes for lignocellulosic ethanol production, and development of an innovative method. Biomass and Bioenergy, v.46, p.25-35, 2012. Elsevier Ltd. Disponível em: < http://linkinghub. elsevier.com/retrieve/pii/S0961953412001912>. Acesso em: 21 de dez 2013.

CONAB - Companhia Nacional de Abastecimento (2011) Disponível em: <http://www.conab.gov.br $>$. Acesso em: 04 jan 2014.

DUFF, S.J.B.; MURRAY, W.D. Bioconversion of forest products industry waste cellulosics to fuel ethanol: a review. Bioresource Technology, v.55, p.1- 33, 1996.

HECTOR, R. Developing Yeast Strains for Biomass-to-Ethanol Production. Disponível em: $<$ http://biomassmagazine.com/articles/1533/developing-yeast-strains-for-biomass-to-ethanol-production> Acesso em: 05 agos. 2013.

HUBER, G.W.; DALE, B.E. Gasolinade capim e outros vegetais. Scientific American Brasil, n.87, 2009.

JR. SELEGHIM, P.; POLIKARPOV, I. Desafios para transformar conceitos em realidade. Scientific American Brasil, n.87, 2009.

JR. PEREIRA, N. Biomass of lignocellulosic composition For fuel ethanol production within the context of biorefinery. Series on Biotechnology, Escola de Química, Universidade Federal do Rio de Janeiro, v.2, p.2-47, 2008.

LEITE, R.C.; CORTEZ, L.A.B. O Etanol Combustível no Brasil. Ministério das Relações Exteriores, Editora Gráfica Ltda, p.61-75, 2007.

LIMAYEM, A.; RICKE, S.C. Lignocellulosic biomass for bioethanol production: Current perspectives, potential issues and future prospects. Progress in Energy and Combustion Science, v. 38, n. 4, p.449-467, 2012.

MARCONATO, M.S.; SANTINI, G.A. Alternativas para a geração de energia renovável no brasil: a opção pela biomassa. In: XLVI CONGRESSO DA SOCIEDADE BRASILEIRA DE ECONOMIA, ADMINISTRAÇÃO E SOCIOLOGIA RURAL, Tupã-SP, 2008.

MARTINS, L.F.; MARTINS, L.F.; KOLLING, D.; CAMASSOLA, M.; DILON, A. J.P.; RAMOS, L.P. Perfil hidrolítico das cellulases de Penicillium echinulatum. In: SIMPÓSIO NACIONAL DE BIOPROCESSOS, Anais do XV Simpósio Nacional de Bioprocessos, Recife, Brasil, 2005.

Petrobrás Biocombustível. Produção comercial de etanol de segunda geração tem meta para 2015. Disponível 
em: $<$ http://fatosedados.blogspetrobras.com.br/2013/03/20/producao-comercial-de-etanol-de-segunda-geracao-tem-meta-para-2015/> Acesso em: $06 \mathrm{dez} .2013$.

ROSSELL, C.E.V. Processo DHR (Dedini Hidrólise Rápida) - projeto, implantação e operação da unidade de desenvolvimento de processo (UDP). Centro de Tecnologia da Cooperativa de Produtores de Cana, Açúcar e Álcool do Estado de São Paulo (Copersucar), 2002.

SANTOS, J.R.A.; MAIOR, A.M.S.; GOUVEIA, E.R.; MARTÍN, C. Comparação entre processos em SHF e em SSF de bagaço de cana-de-açúcar para a produção de etanol por Saccharomyces cerevisiae. Química Nova, v.33, n.4, 2010.

SANTOS, J.R.A.; MAIOR-SOUTO, M.; GOUVEIA, E.R. Comparação entre processos em SHF e em SSF de bagaço de cana-de-açucar para a produção de etanol por Saccharomyces cerevisiae. Departamento de Antibióticos, Universidade Federal de Pernambuco, Recife - PE, Brasil, 2009.

SANTOS, F.A.; QUEIRÓZ, J.H. DE; COLODETTE, J.L.; FERNANDES, S.A.; GUIMARÃES, V.M. Potemcial da palha de cana-de-aucar para produção de etanol. Quimica nova, v.35, n.5, p.1004-1010, 2012.

SAUER. Biocombustíveis no Brasil comercialização e logística.Biocombustíveis no Brasil realidades e perspectivas. Ministério da Relações Exteriores, Editora Gráfica Ltda, 2007.

SUN, Y.; CHENG, J.J. Dilute acid pretreatment of rye straw and bermudagrass for ethaol production. Bioresource Technology, v.96, p.1599-1606, 2005.

TRIGUEIRO, A. Etanol de segunda geração se prepara para entrar no mercado. Disponível em: $<\underline{\text { http:// }}$ g1.globo.com/jornal-da-globo/noticia/2013/05/etanol-de-segunda-geracao-se-prepara-para-entrar-no-mercado.html>Acesso em: 16 out. 2013.

WORLD WATCH INSTITUTE. Biofuels for transportation. Gobal potential and implicatios for sustainable agriculture and energy in the 21st century, Extended summary, 2006. 